\title{
Search for Pair Production of Doubly Charged Higgs Bosons in the $\boldsymbol{H}^{++} \boldsymbol{H}^{--} \rightarrow \boldsymbol{\mu}^{+} \boldsymbol{\mu}^{+} \boldsymbol{\mu}^{-} \boldsymbol{\mu}^{-}$Final State
}

V. M. Abazov,${ }^{36}$ B. Abbott,${ }^{75}$ M. Abolins, ${ }^{65}$ B. S. Acharya,${ }^{29}$ M. Adams,${ }^{51}$ T. Adams,${ }^{49}$ E. Aguilo, ${ }^{6}$ S. H. Ahn, ${ }^{31}$ M. Ahsan, ${ }^{59}$ G. D. Alexeev ${ }^{36}$ G. Alkhazov,${ }^{40}$ A. Alton, ${ }^{64, *}$ G. Alverson, ${ }^{63}$ G. A. Alves, ${ }^{2}$ M. Anastasoaie, ${ }^{35}$ L. S. Ancu, ${ }^{35}$ T. Andeen ${ }^{53}$ S. Anderson, ${ }^{45}$ B. Andrieu, ${ }^{17}$ M. S. Anzelc, ${ }^{53}$ M. Aoki,${ }^{50}$ Y. Arnoud, ${ }^{14}$ M. Arov, ${ }^{60}$ M. Arthaud, ${ }^{18}$ A. Askew, ${ }^{49}$ B. Åsman, ${ }^{41}$ A. C. S. Assis Jesus, ${ }^{3}$ O. Atramentov, ${ }^{49}$ C. Avila, ${ }^{8}$ C. Ay, ${ }^{24}$ F. Badaud, ${ }^{13}$ A. Baden,${ }^{61}$ L. Bagby, ${ }^{50}$ B. Baldin,${ }^{50}$ D. V. Bandurin, ${ }^{59}$ P. Banerjee, ${ }^{29}$ S. Banerjee, ${ }^{29}$ E. Barberis, ${ }^{63}$ A.-F. Barfuss, ${ }^{15}$ P. Bargassa,${ }^{80}$ P. Baringer, ${ }^{58}$ J. Barreto, ${ }^{2}$

J. F. Bartlett, ${ }^{50}$ U. Bassler, ${ }^{18}$ D. Bauer, ${ }^{43}$ S. Beale, ${ }^{6}$ A. Bean,${ }^{58}$ M. Begalli, ${ }^{3}$ M. Begel,${ }^{73}$ C. Belanger-Champagne, ${ }^{41}$ L. Bellantoni, ${ }^{50}$ A. Bellavance,${ }^{50}$ J. A. Benitez, ${ }^{65}$ S. B. Beri, ${ }^{27}$ G. Bernardi,${ }^{17}$ R. Bernhard,${ }^{23}$ I. Bertram,${ }^{42}$ M. Besançon, ${ }^{18}$ R. Beuselinck, ${ }^{43}$ V. A. Bezzubov, ${ }^{39}$ P. C. Bhat,${ }^{50}$ V. Bhatnagar ${ }^{27}$ C. Biscarat,${ }^{20}$ G. Blazey,${ }^{52}$ F. Blekman, ${ }^{43}$ S. Blessing, ${ }^{49}$ D. Bloch,${ }^{19}$ K. Bloom, ${ }^{67}$ A. Boehnlein, ${ }^{50}$ D. Boline, ${ }^{62}$ T. A. Bolton, ${ }^{59}$ G. Borissov,${ }^{42}$ T. Bose, ${ }^{77}$ A. Brandt, ${ }^{78}$ R. Brock, ${ }^{65}$

G. Brooijmans ${ }^{70}$ A. Bross ${ }^{50}$ D. Brown, ${ }^{81}$ N. J. Buchanan, ${ }^{49}$ D. Buchholz, ${ }^{53}$ M. Buehler,${ }^{81}$ V. Buescher,${ }^{22}$ V. Bunichev, ${ }^{38}$ S. Burdin, ${ }^{42,+}$ S. Burke, ${ }^{45}$ T. H. Burnett, ${ }^{82}$ C. P. Buszello, ${ }^{43}$ J. M. Butler, ${ }^{62}$ P. Calfayan, ${ }^{25}$ S. Calvet, ${ }^{16}$ J. Cammin, ${ }^{71}$ W. Carvalho, ${ }^{3}$ B. C. K. Casey,${ }^{50}$ H. Castilla-Valdez,${ }^{33}$ S. Chakrabarti, ${ }^{18}$ D. Chakraborty, ${ }^{52}$ K. Chan, ${ }^{6}$ K. M. Chan, ${ }^{55}$ A. Chandra, ${ }^{48}$ F. Charles,${ }^{19}$ E. Cheu ${ }^{45}$ F. Chevallier, ${ }^{14}$ D. K. Cho, ${ }^{62}$ S. Choi,${ }^{32}$ B. Choudhary, ${ }^{28}$ L. Christofek, ${ }^{77}$ T. Christoudias, ${ }^{43}$ S. Cihangir, ${ }^{50}$ D. Claes,${ }^{67}$ Y. Coadou, ${ }^{6}$ M. Cooke, ${ }^{80}$ W. E. Cooper, ${ }^{50}$ M. Corcoran, ${ }^{80}$ F. Couderc, ${ }^{18}$ M.-C. Cousinou, ${ }^{15}$ S. Crépé-Renaudin, ${ }^{14}$ D. Cutts, ${ }^{77}$ M. Ćwiok,${ }^{30}$ H. da Motta, ${ }^{2}$ A. Das, ${ }^{45}$ G. Davies,${ }^{43}$ K. De,${ }^{78}$ S. J. de Jong, ${ }^{35}$ E. De La Cruz-Burelo,${ }^{64}$ C. De Oliveira Martins, ${ }^{3}$ J. D. Degenhardt, ${ }^{64}$ F. Déliot, ${ }^{18}$ M. Demarteau, ${ }^{50}$ R. Demina, ${ }^{71}$ D. Denisov, ${ }^{50}$ S. P. Denisov, ${ }^{39}$ S. Desai,${ }^{50}$ H. T. Diehl,${ }^{50}$ M. Diesburg, ${ }^{50}$ A. Dominguez, ${ }^{67}$ H. Dong,${ }^{72}$ L. V. Dudko, ${ }^{38}$ L. Duflot, ${ }^{16}$ S. R. Dugad,${ }^{29}$ D. Duggan ${ }^{49}$ A. Duperrin, ${ }^{15}$ J. Dyer, ${ }^{65}$ A. Dyshkant, ${ }^{52}$ M. Eads,${ }^{67}$ D. Edmunds, ${ }^{65}$ J. Ellison, ${ }^{48}$ V. D. Elvira, ${ }^{50}$ Y. Enari, ${ }^{77}$ S. Eno,${ }^{61}$ P. Ermolov,${ }^{38}$ H. Evans,${ }^{54}$ A. Evdokimov, ${ }^{73}$ V. N. Evdokimov ${ }^{39}$ A. V. Ferapontov, ${ }^{59}$ T. Ferbel, ${ }^{71}$ F. Fiedler, ${ }^{24}$ F. Filthaut, ${ }^{35}$ W. Fisher, ${ }^{50}$ H. E. Fisk, ${ }^{50}$ M. Fortner,${ }^{52}$ H. Fox,${ }^{42}$ S. Fu, ${ }^{50}$ S. Fuess,${ }^{50}$ T. Gadfort, ${ }^{70}$ C. F. Galea, ${ }^{35}$ E. Gallas, ${ }^{50}$ C. Garcia, ${ }^{71}$ A. Garcia-Bellido, ${ }^{82}$ V. Gavrilov, ${ }^{37}$ P. Gay, ${ }^{13}$ W. Geist ${ }^{19}$ D. Gelé, ${ }^{19}$ C. E. Gerber, ${ }^{51}$ Y. Gershtein, ${ }^{49}$ D. Gillberg, ${ }^{6}$ G. Ginther, ${ }^{71}$ N. Gollub, ${ }^{41}$ B. Gómez, ${ }^{8}$

A. Goussiou, ${ }^{82}$ P. D. Grannis, ${ }^{72}$ H. Greenlee, ${ }^{50}$ Z. D. Greenwood ${ }^{60}$ E. M. Gregores ${ }^{4}$ G. Grenier, ${ }^{20}$ Ph. Gris, ${ }^{13}$ J.-F. Grivaz, ${ }^{16}$ A. Grohsjean, ${ }^{25}$ S. Grünendahl,${ }^{50}$ M. W. Grünewald ${ }^{30}$ F. Guo,${ }^{72}$ J. Guo ${ }^{72}$ G. Gutierrez,${ }^{50}$ P. Gutierrez, ${ }^{75}$ A. Haas, ${ }^{70}$ N. J. Hadley,${ }^{61}$ P. Haefner, ${ }^{25}$ S. Hagopian, ${ }^{49}$ J. Haley, ${ }^{68}$ I. Hall, ${ }^{65}$ R. E. Hall, ${ }^{47}$ L. Han,${ }^{7}$ K. Harder,${ }^{44}$ A. Harel, ${ }^{71}$ R. Harrington, ${ }^{63}$ J. M. Hauptman, ${ }^{57}$ R. Hauser, ${ }^{65}$ J. Hays, ${ }^{43}$ T. Hebbeker, ${ }^{21}$ D. Hedin,${ }^{52}$ J. G. Hegeman, ${ }^{34}$

J. M. Heinmiller, ${ }^{51}$ A. P. Heinson, ${ }^{48}$ U. Heintz, ${ }^{62}$ C. Hensel, ${ }^{58}$ K. Herner, ${ }^{72}$ G. Hesketh, ${ }^{63}$ M. D. Hildreth, ${ }^{55}$ R. Hirosky, ${ }^{81}$ J. D. Hobbs, ${ }^{72}$ B. Hoeneisen, ${ }^{12}$ H. Hoeth, ${ }^{26}$ M. Hohlfeld, ${ }^{22}$ S. J. Hong, ${ }^{31}$ S. Hossain,${ }^{75}$ P. Houben, ${ }^{34}$ Y. Hu, ${ }^{72}$ Z. Hubacek, ${ }^{10}$ V. Hynek, ${ }^{9}$ I. Iashvili, ${ }^{69}$ R. Illingworth, ${ }^{50}$ A. S. Ito ${ }^{50}$ S. Jabeen, ${ }^{62}$ M. Jaffré,${ }^{16}$ S. Jain,${ }^{75}$ K. Jakobs, ${ }^{23}$ C. Jarvis, ${ }^{61}$ R. Jesik, ${ }^{43}$

K. Johns,${ }^{45}$ C. Johnson, ${ }^{70}$ M. Johnson,${ }^{50}$ A. Jonckheere, ${ }^{50}$ P. Jonsson, ${ }^{43}$ A. Juste, ${ }^{50}$ E. Kajfasz, ${ }^{15}$ A. M. Kalinin,${ }^{36}$ J. M. Kalk, ${ }^{60}$ S. Kappler, ${ }^{21}$ D. Karmanov, ${ }^{38}$ P. A. Kasper,${ }^{50}$ I. Katsanos, ${ }^{70}$ D. Kau, ${ }^{49}$ V. Kaushik, ${ }^{78}$ R. Kehoe, ${ }^{79}$ S. Kermiche, ${ }^{15}$ N. Khalatyan, ${ }^{50}$ A. Khanov ${ }^{76}$ A. Kharchilava, ${ }^{69}$ Y. M. Kharzheev, ${ }^{36}$ D. Khatidze, ${ }^{70}$ T. J. Kim, ${ }^{31}$ M. H. Kirby, ${ }^{53}$ M. Kirsch ${ }^{21}$ B. Klima,${ }^{50}$ J. M. Kohli, ${ }^{27}$ J.-P. Konrath, ${ }^{23}$ V. M. Korablev ${ }^{39}$ A. V. Kozelov, ${ }^{39}$ J. Kraus, ${ }^{65}$ D. Krop, ${ }^{54}$ T. Kuhl,${ }^{24}$ A. Kumar, ${ }^{69}$ A. Kupco, ${ }^{11}$ T. Kurča, ${ }^{20}$ J. Kvita, ${ }^{9}$ F. Lacroix,${ }^{13}$ D. Lam, ${ }^{55}$ S. Lammers,${ }^{70}$ G. Landsberg, ${ }^{77}$ P. Lebrun, ${ }^{20}$ W. M. Lee,${ }^{50}$ A. Leflat,${ }^{38}$ J. Lellouch, ${ }^{17}$ J. Leveque,${ }^{45}$ J. Li, ${ }^{78}$ L. Li,${ }^{48}$ Q. Z. Li,${ }^{50}$ S. M. Lietti, ${ }^{5}$ J. G. R. Lima ${ }^{52}$ D. Lincoln, ${ }^{50}$ J. Linnemann, ${ }^{65}$ V. V. Lipaev, ${ }^{39}$ R. Lipton, ${ }^{50}$ Y. Liu, ${ }^{7}$ Z. Liu, ${ }^{6}$ A. Lobodenko, ${ }^{40}$

M. Lokajicek, ${ }^{11}$ P. Love, ${ }^{42}$ H. J. Lubatti, ${ }^{82}$ R. Luna, ${ }^{3}$ A. L. Lyon, ${ }^{50}$ A. K. A. Maciel, ${ }^{2}$ D. Mackin, ${ }^{80}$ R. J. Madaras, ${ }^{46}$ P. Mättig, ${ }^{26}$ C. Magass, ${ }^{21}$ A. Magerkurth, ${ }^{64}$ P. K. Mal, ${ }^{82}$ H. B. Malbouisson, ${ }^{3}$ S. Malik, ${ }^{67}$ V. L. Malyshev, ${ }^{36}$ H. S. Mao, ${ }^{50}$ Y. Maravin, ${ }^{59}$ B. Martin, ${ }^{14}$ R. McCarthy, ${ }^{72}$ A. Melnitchouk, ${ }^{66}$ L. Mendoza, ${ }^{8}$ P. G. Mercadante, ${ }^{5}$ M. Merkin, ${ }^{88}$

K. W. Merritt, ${ }^{50}$ A. Meyer, ${ }^{21}$ J. Meyer, ${ }^{22,}{ }^{\text {T. Millet }}{ }^{20}$ J. Mitrevski, ${ }^{70}$ J. Molina, ${ }^{3}$ R. K. Mommsen, ${ }^{44}$ N. K. Mondal, ${ }^{29}$

R. W. Moore, ${ }^{6}$ T. Moulik,${ }^{58}$ G. S. Muanza, ${ }^{20}$ M. Mulders,${ }^{50}$ M. Mulhearn,${ }^{70}$ O. Mundal,${ }^{22}$ L. Mundim, ${ }^{3}$ E. Nagy, ${ }^{15}$ M. Naimuddin, ${ }^{50}$ M. Narain, ${ }^{77}$ N. A. Naumann, ${ }^{35}$ H. A. Neal, ${ }^{64}$ J.P. Negret, ${ }^{8}$ P. Neustroev, ${ }^{40}$ H. Nilsen,${ }^{23}$ H. Nogima, ${ }^{3}$ S. F. Novaes, ${ }^{5}$ T. Nunnemann, ${ }^{25}$ V. O’Dell, ${ }^{50}$ D. C. O'Neil,${ }^{6}$ G. Obrant, ${ }^{40}$ C. Ochando, ${ }^{16}$ D. Onoprienko, ${ }^{59}$ N. Oshima, ${ }^{50}$ N. Osman, ${ }^{43}$ J. Osta ${ }^{55}$ R. Otec, ${ }^{10}$ G. J. Otero y Garzón, ${ }^{50}$ M. Owen, ${ }^{44}$ P. Padley,${ }^{80}$ M. Pangilinan, ${ }^{77}$ N. Parashar, ${ }^{56}$ S.-J. Park ${ }^{71}$ S. K. Park,${ }^{31}$ J. Parsons,${ }^{70}$ R. Partridge,${ }^{77}$ N. Parua,${ }^{54}$ A. Patwa ${ }^{73}$ G. Pawloski ${ }^{80}$ B. Penning,${ }^{23}$ M. Perfilov, ${ }^{38}$ K. Peters, ${ }^{44}$ Y. Peters,${ }^{26}$ P. Pétroff, ${ }^{16}$ M. Petteni, ${ }^{43}$ R. Piegaia, ${ }^{1}$ J. Piper,${ }^{65}$ M.-A. Pleier, ${ }^{22}$ P. L. M. Podesta-Lerma, ${ }^{33,}$ V. M. Podstavkov, ${ }^{50}$ Y. Pogorelov, ${ }^{55}$ M.-E. Pol,,${ }^{2}$ P. Polozov, ${ }^{37}$ B. G. Pope,${ }^{65}$ A. V. Popov, ${ }^{39}$ C. Potter,${ }^{6}$ 
W. L. Prado da Silva, ${ }^{3}$ H. B. Prosper, ${ }^{49}$ S. Protopopescu, ${ }^{73}$ J. Qian,${ }^{64}$ A. Quadt ${ }^{22,8}$ B. Quinn, ${ }^{66}$ A. Rakitine, ${ }^{42}$ M. S. Rangel, ${ }^{2}$ K. Ranjan, ${ }^{28}$ P. N. Ratoff, ${ }^{42}$ P. Renkel, ${ }^{79}$ S. Reucroft,${ }^{63}$ P. Rich, ${ }^{44}$ J. Rieger, ${ }^{54}$ M. Rijssenbeek, ${ }^{72}$ I. Ripp-Baudot, ${ }^{19}$ F. Rizatdinova ${ }^{76}$ S. Robinson, ${ }^{43}$ R. F. Rodrigues, ${ }^{3}$ M. Rominsky, ${ }^{75}$ C. Royon,${ }^{18}$ P. Rubinov, ${ }^{50}$ R. Ruchti, ${ }^{55}$ G. Safronov, ${ }^{37}$ G. Sajot,${ }^{14}$ A. Sánchez-Hernández,${ }^{33}$ M. P. Sanders, ${ }^{17}$ A. Santoro, ${ }^{3}$ G. Savage, ${ }^{50}$ L. Sawyer,${ }^{60}$ T. Scanlon, ${ }^{43}$ D. Schaile, ${ }^{25}$ R. D. Schamberger, ${ }^{72}$ Y. Scheglov, ${ }^{40}$ H. Schellman, ${ }^{53}$ T. Schliephake, ${ }^{26}$ C. Schwanenberger ${ }^{44}$

A. Schwartzman, ${ }^{68}$ R. Schwienhorst ${ }^{65}$ J. Sekaric, ${ }^{49}$ H. Severini, ${ }^{75}$ E. Shabalina, ${ }^{51}$ M. Shamim, ${ }^{59}$ V. Shary, ${ }^{18}$ A. A. Shchukin, ${ }^{39}$ R. K. Shivpuri, ${ }^{28}$ V. Siccardi, ${ }^{19}$ V. Simak, ${ }^{10}$ V. Sirotenko, ${ }^{50}$ P. Skubic, ${ }^{75}$ P. Slattery, ${ }^{71}$ D. Smirnov, ${ }^{55}$ G. R. Snow, ${ }^{67}$ J. Snow, ${ }^{74}$ S. Snyder, ${ }^{73}$ S. Söldner-Rembold, ${ }^{44}$ L. Sonnenschein, ${ }^{17}$ A. Sopczak, ${ }^{42}$ M. Sosebee,${ }^{78}$ K. Soustruznik, ${ }^{9}$ B. Spurlock, ${ }^{78}$ J. Stark,,${ }^{14}$ J. Steele,${ }^{60}$ V. Stolin, ${ }^{37}$ D. A. Stoyanova, ${ }^{39}$ J. Strandberg, ${ }^{64}$ S. Strandberg, ${ }^{41}$ M. A. Strang, ${ }^{69}$ E. Strauss, ${ }^{72}$ M. Strauss, ${ }^{75}$ R. Ströhmer, ${ }^{25}$ D. Strom,${ }^{53}$ L. Stutte,${ }^{50}$ S. Sumowidagdo, ${ }^{49}$ P. Svoisky, ${ }^{55}$ A. Sznajder, ${ }^{3}$ P. Tamburello, ${ }^{45}$ A. Tanasijczuk, ${ }^{1}$ W. Taylor, ${ }^{6}$ J. Temple, ${ }^{45}$ B. Tiller, ${ }^{25}$ F. Tissandier ${ }^{13}$ M. Titov, ${ }^{18}$ V. V. Tokmenin ${ }^{36}$ T. Toole, ${ }^{61}$ I. Torchiani, ${ }^{23}$ T. Trefzger, ${ }^{24}$ D. Tsybychev,${ }^{72}$ B. Tuchming,${ }^{18}$ C. Tully, ${ }^{68}$ P. M. Tuts, ${ }^{70}$ R. Unalan, ${ }^{65}$ L. Uvarov, ${ }^{40}$ S. Uvarov ${ }^{40}$ S. Uzunyan, ${ }^{52}$ B. Vachon, ${ }^{6}$ P. J. van den Berg, ${ }^{34}$ R. Van Kooten, ${ }^{54}$ W. M. van Leeuwen, ${ }^{34}$ N. Varelas, ${ }^{51}$ E. W. Varnes ${ }^{45}$ I. A. Vasilyev, ${ }^{39}$ M. Vaupel,${ }^{26}$ P. Verdier, ${ }^{20}$ L. S. Vertogradov, ${ }^{36}$ M. Verzocchi, ${ }^{50}$ F. Villeneuve-Seguier, ${ }^{43}$ P. Vint,${ }^{43}$ P. Vokac,${ }^{10}$ E. Von Toerne, ${ }^{59} \mathrm{M}$. Voutilainen, ${ }^{68, \|}$ R. Wagner,${ }^{68}$ H. D. Wahl, ${ }^{49}$ L. Wang, ${ }^{61}$ M. H.L. S. Wang, ${ }^{50}$ J. Warchol,${ }^{55}$ G. Watts, ${ }^{82}$ M. Wayne, ${ }^{55}$ G. Weber, ${ }^{24}$ M. Weber, ${ }^{50}$ L. Welty-Rieger, ${ }^{54}$ A. Wenger, ${ }^{23, \pi} \mathrm{N}$. Wermes ${ }^{22} \mathrm{M}$. Wetstein, ${ }^{61}$ A. White,${ }^{78} \mathrm{D}$. Wicke, ${ }^{26} \mathrm{G}$. W. Wilson, ${ }^{58}$ S. J. Wimpenny, ${ }^{48}$ M. Wobisch, ${ }^{60}$ D. R. Wood, ${ }^{63}$ T. R. Wyatt, ${ }^{44}$ Y. Xie, ${ }^{77}$ S. Yacoob,${ }^{53}$ R. Yamada, ${ }^{50}$ M. Yan,${ }^{61}$ T. Yasuda,${ }^{50}$ Y. A. Yatsunenko, ${ }^{36}$ K. Yip, ${ }^{73}$ H. D. Yoo, ${ }^{77}$ S. W. Youn, ${ }^{53}$ J. Yu, ${ }^{78}$ A. Zatserklyaniy, ${ }^{52}$ C. Zeitnitz, ${ }^{26}$ T. Zhao,${ }^{82}$ B. Zhou, ${ }^{64}$ J. Zhu, ${ }^{72}$ M. Zielinski, ${ }^{71}$ D. Zieminska, ${ }^{54}$ A. Zieminski, ${ }^{54, * *}$ L. Zivkovic, ${ }^{70}$ V. Zutshi, ${ }^{52}$ and E. G. Zverev ${ }^{38}$

\title{
(D0 Collaboration)
}

\author{
${ }^{1}$ Universidad de Buenos Aires, Buenos Aires, Argentina \\ ${ }^{2}$ LAFEX, Centro Brasileiro de Pesquisas Físicas, Rio de Janeiro, Brazil \\ ${ }^{3}$ Universidade do Estado do Rio de Janeiro, Rio de Janeiro, Brazil \\ ${ }^{4}$ Universidade Federal do ABC, Santo André, Brazil \\ ${ }^{5}$ Instituto de Física Teórica, Universidade Estadual Paulista, São Paulo, Brazil \\ ${ }^{6}$ University of Alberta, Edmonton, Alberta, Canada, \\ Simon Fraser University, Burnaby, British Columbia, Canada, \\ York University, Toronto, Ontario, Canada, \\ and McGill University, Montreal, Quebec, Canada \\ ${ }^{7}$ University of Science and Technology of China, Hefei, People's Republic of China \\ ${ }^{8}$ Universidad de los Andes, Bogotá, Colombia \\ ${ }^{9}$ Center for Particle Physics, Charles University, Prague, Czech Republic \\ ${ }^{10}$ Czech Technical University, Prague, Czech Republic \\ ${ }^{11}$ Center for Particle Physics, Institute of Physics, Academy of Sciences of the Czech Republic, Prague, Czech Republic \\ ${ }^{12}$ Universidad San Francisco de Quito, Quito, Ecuador \\ ${ }^{13}$ LPC, Université Blaise Pascal, CNRS/IN2P3, Clermont, France \\ ${ }^{14}$ LPSC, Université Joseph Fourier Grenoble 1, CNRS/IN2P3, Institut National Polytechnique de Grenoble, France \\ ${ }^{15}$ CPPM, IN2P3/CNRS, Université de la Méditerranée, Marseille, France \\ ${ }^{16}$ LAL, Université Paris-Sud, IN2P3/CNRS, Orsay, France \\ ${ }^{17}$ LPNHE, IN2P3/CNRS, Universités Paris VI and VII, Paris, France \\ ${ }^{18}$ DAPNIA/Service de Physique des Particules, CEA, Saclay, France \\ ${ }^{19}$ IPHC, Université Louis Pasteur et Université de Haute Alsace, CNRS/IN2P3, Strasbourg, France \\ ${ }^{20} I P N L$, Université Lyon 1, CNRS/IN2P3, Villeurbanne, France, and \\ Université de Lyon, Lyon, France \\ ${ }^{21}$ III. Physikalisches Institut A, RWTH Aachen, Aachen, Germany \\ ${ }^{22}$ Physikalisches Institut, Universität Bonn, Bonn, Germany \\ ${ }^{23}$ Physikalisches Institut, Universität Freiburg, Freiburg, Germany \\ ${ }^{24}$ Institut für Physik, Universität Mainz, Mainz, Germany \\ ${ }^{25}$ Ludwig-Maximilians-Universität München, München, Germany \\ ${ }^{26}$ Fachbereich Physik, University of Wuppertal, Wuppertal, Germany \\ ${ }^{27}$ Panjab University, Chandigarh, India \\ ${ }^{28}$ Delhi University, Delhi, India \\ ${ }^{29}$ Tata Institute of Fundamental Research, Mumbai, India
}




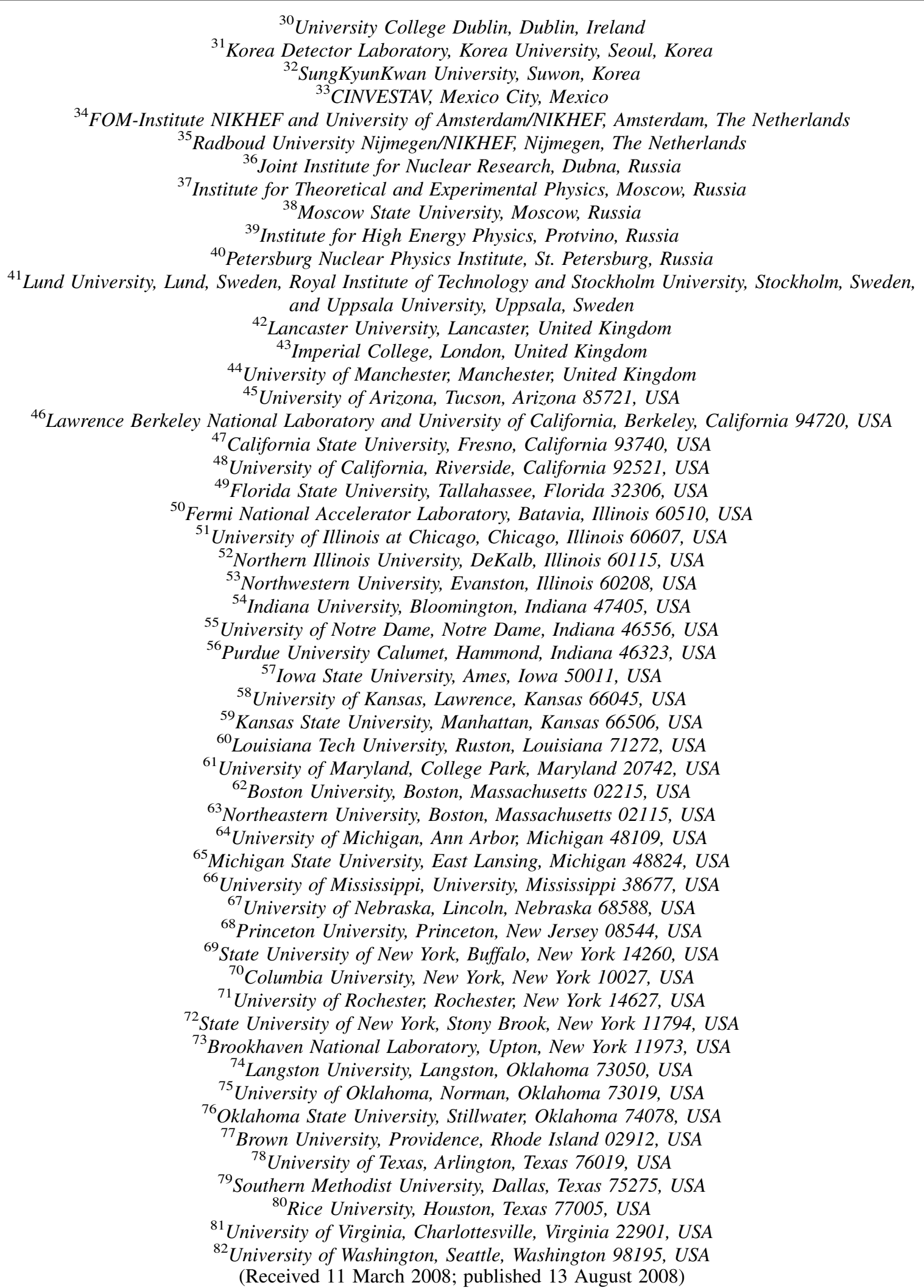

We report the results of a search for pair production of doubly charged Higgs bosons via $p \bar{p} \rightarrow$ $H^{++} H^{--} X \rightarrow \mu^{+} \mu^{+} \mu^{-} \mu^{-} X$ at $\sqrt{s}=1.96 \mathrm{TeV}$. We use a data set corresponding to an integrated luminosity of $1.1 \mathrm{fb}^{-1}$ collected from 2002 to 2006 by the D0 detector at the Fermilab Tevatron Collider. In the absence of an excess above the standard model background, lower mass limits of $M\left(H_{L}^{ \pm \pm}\right)>150 \mathrm{GeV} / c^{2}$ 
and $M\left(H_{R}^{ \pm \pm}\right)>127 \mathrm{GeV} / c^{2}$ at $95 \%$ C.L. are set, respectively, for left-handed and right-handed doubly charged Higgs bosons assuming a $100 \%$ branching ratio into muons.

In the standard model (SM) of electroweak interactions, elementary fermions and bosons acquire mass via a weak isospin scalar doublet. This mechanism results in the existence of an additional particle, the Higgs boson, which has not yet been observed. Extensions of the Higgs sector involving higher isospin multiplets predict the existence of doubly charged Higgs bosons, which can be relatively light and hence accessible at current experimental facilities. Doubly charged Higgs bosons appear in many scenarios such as left-right symmetric models [1], Higgs triplet models [2], and little Higgs models [3]. At the Fermilab Tevatron Collider, the two main production mechanisms are pair production via $p \bar{p} \rightarrow Z / \gamma^{*} X \rightarrow H^{++} H^{--} X$ and single production via $W W$ fusion, $p \bar{p} \rightarrow W^{ \pm} W^{ \pm} X \rightarrow$ $H^{ \pm \pm} X$. However, higher isospin Higgs multiplets are generally severely constrained by $\rho \equiv m_{W}^{2} /\left(\cos \theta_{W} m_{Z}\right)^{2}=1$ at tree level. The existing phenomenological and theoretical constraints are easily satisfied when the $W^{ \pm} W^{ \pm} \rightarrow$ $\mathrm{H}^{ \pm \pm}$coupling is vanishing [4]. If the $\mathrm{H}^{++}$coupling to $W$ boson pairs is suppressed, the dominant final states are expected to be like-sign lepton pairs. Left-handed $\left(H_{L}^{ \pm \pm}\right)$ and right-handed $\left(H_{R}^{ \pm \pm}\right)$states are distinguished by their coupling to left-handed and right-handed leptons, respectively. While there is a prejudice that decays to heavier leptons would be favored, the relative branching ratios into different lepton flavors cannot be predicted by the theory. Therefore, it is customary to consider benchmark scenarios assuming a $100 \%$ branching ratio into a particular lepton flavor. The pair production cross section for left-handed doubly charged Higgs bosons for $100 \leq M\left(H^{ \pm \pm}\right) \leq$ $200 \mathrm{GeV} / c^{2}$ is about a factor of 2 larger than that for the right-handed states due to different couplings to the intermediate $Z$ boson [5]. Previous searches for $H^{ \pm \pm}$have been performed by the LEP Collaborations [6] in $e^{+} e^{-}$collisions and by the D0 [7] and CDF [8] Collaborations at the Tevatron $p \bar{p}$ collider. This Letter presents the results of a direct search for $p \bar{p} \rightarrow H^{++} H^{--} X$ with $H^{ \pm \pm} \rightarrow \mu^{ \pm} \mu^{ \pm}$ by the D0 Collaboration with improved sensitivity.

The main D0 detector systems are a central tracking system, a liquid-argon and uranium calorimeter, and a muon detector [9]. The central tracking system consists of the silicon microstrip tracker (SMT) and the central fiber tracker (CFT) surrounded by a $2 \mathrm{~T}$ solenoidal magnet, with designs optimized for tracking and vertexing capability at pseudorapidity [10] $|\eta|<3$ and $|\eta|<2.5$, respectively. The liquid-argon and uranium calorimeter has a central calorimeter covering a region up to $|\eta| \approx 1.1$ and two end calorimeters extending the coverage to $|\eta| \approx 4.2$ [11]. The muon detector has layers of proportional drift tubes and scintillation counters before and after a $1.8 \mathrm{~T}$ iron toroid
[12]. This analysis is based on the run II data set collected with the D0 detector at $\sqrt{s}=1.96 \mathrm{TeV}$ from April 2002 to February 2006 corresponding to $1.1 \mathrm{fb}^{-1}$. Events are collected using a suite of dimuon and single muon triggers.

In the previous D0 analysis [7], two like-sign muons were required in the final state. In this analysis, we require a third muon, which increases the sensitivity by decreasing backgrounds. We follow five steps to select events. In the first step $(S 1)$, events are required to have at least two muons. Each muon must have a transverse momentum $p_{T}>15 \mathrm{GeV} / c$ and $|\eta|<2.0$. Muons are selected using patterns of hits in the wire chambers and scintillators in the muon system. Each muon must be matched to a track in the
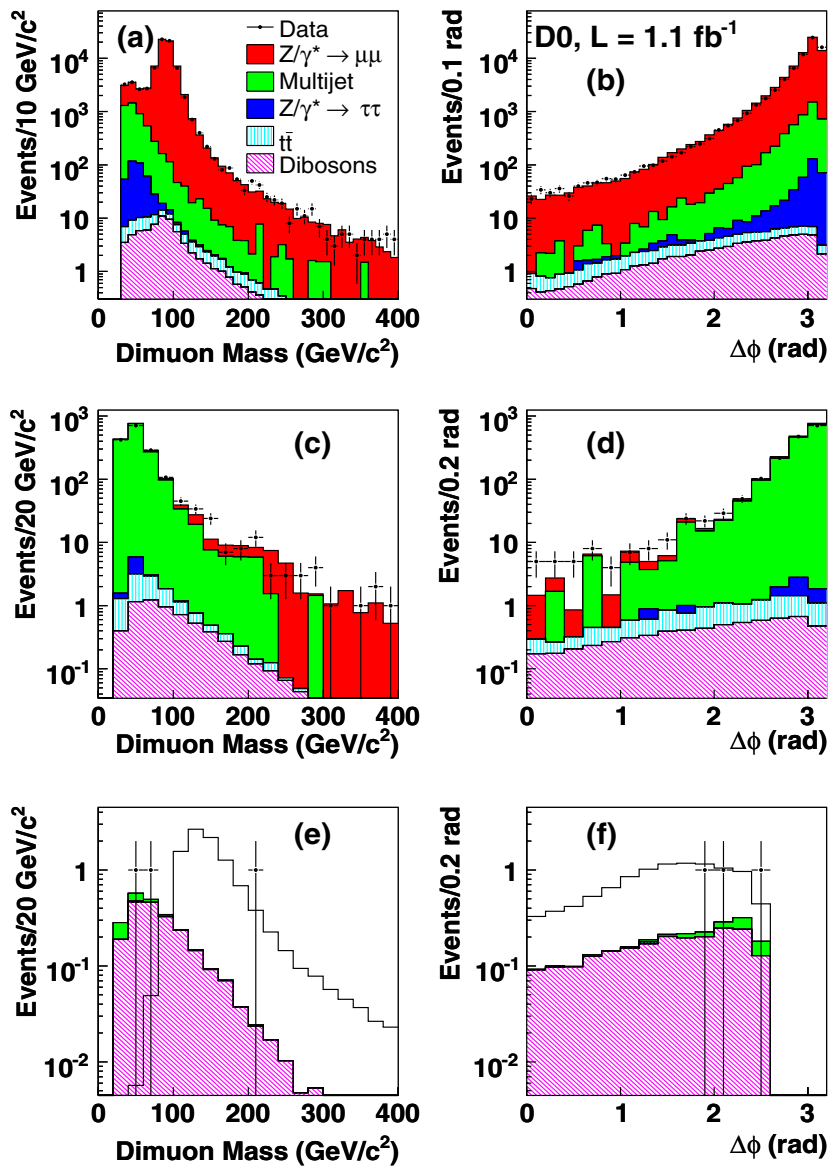

FIG. 1 (color online). Distributions of the dimuon invariant mass and $\Delta \phi$ between the two muons for data compared to the sum of MC backgrounds after selection $S 1$ (a),(b), the preselection $S 1$ with the like-sign requirement $S 4$ (c),(d), and the final requirement $S 1-S 5$ (e),(f). The signal expected for a left-handed $H^{ \pm \pm}$, with $M\left(H^{ \pm \pm}\right)=140 \mathrm{GeV} / c^{2}$, is also shown by the open histogram (e),(f). 
central tracker with at least five hits in the CFT layers and at least two hits in the SMT layers. Muons from cosmic rays are removed by using a timing information on the hits in the scintillator layers.

In the second step $(S 2)$, isolation criteria based on the calorimeter and tracking information are applied to remove the background from multijet production with muons originating from in-flight decay of pions or kaons or from semileptonic decays of $B$ or $D$ mesons. The sum of the transverse energies of the calorimeter cells in an annulus of radius $0.1<\mathcal{R}<0.4$, where $\mathcal{R}=\sqrt{(\Delta \phi)^{2}+(\Delta \eta)^{2}}$ and $\phi$ is the azimuthal angle, around the muon direction is required to be less than $2.5 \mathrm{GeV}$. A similar condition is defined for the scalar sum of the $p_{T}$ of all tracks, excluding the muon in a cone of radius $\mathcal{R}=0.5$ centered around the muon, which must be less than $2.5 \mathrm{GeV} / c$.

Selection $S 3$ reduces the remaining $Z \rightarrow \mu^{+} \mu^{-}$and multijet backgrounds. The azimuthal angle $\Delta \phi$ between at least one pair of muons is required to be less than 2.5 radians, since the two muons from $Z$ boson decays are mostly back-to-back. This requirement also rejects a fraction of the multijet background with nearly back-toback muons.

Selection $S 4$ requires at least two muons to be of like sign. The final selection (S5) requires a third muon, satisfying the $S 1$ selection and the isolation selection criteria $S 2$ but without the minimum hit requirement on the central track.

The dominant background in this analysis arises from electroweak processes where real high $p_{T}$ muons are created from $W$ or $Z$ boson decays as well as nonisolated muons originating from jets. The SM backgrounds and signal processes are generated with PYTHIA [13] and normalized using the theoretical cross section. The $Z / \gamma^{*} \rightarrow$ $\ell^{+} \ell^{-}$cross section is calculated at next-to-next-to-leading order (NNLO) [14]. The $t \bar{t}$ cross section is calculated at NNLO [15] and the $W W, Z Z$, and $W Z$ cross sections are calculated with MCFM [16] at next-to-leading order (NLO).
All samples are processed through the D0 detector simulation based on GEANT [17] and the same reconstruction software as for the data. The muon reconstruction and isolation efficiencies differ between Monte Carlo (MC) simulations and data, and these differences are corrected. Trigger efficiency corrections are not applied to the MC samples. Instead, the MC samples are normalized to the data using the $Z$ boson mass peak at the selection level $S 2$.

Another important background comes from multijet production, mainly $b \bar{b}$ events decaying semileptonically into muons that appear isolated. The multijet background is derived from the data sample with nonisolated muons obtained by inverting the isolation requirements for both muons after selection $S 1$. The efficiency of the isolation requirement is assumed to be identical for multijet events with like-sign and opposite-sign muon pairs. It is also assumed that all like-sign events after subtracting SM backgrounds are multijet events. The SM backgrounds are subtracted in the following samples used for the multijet background determination. The total number of multijet events before the isolation requirement $(4492 \pm 120)$ is then given by the number of nonisolated events for all charge combinations multiplied by the ratio of the total number of events to the number of nonisolated events in the like-sign sample. The number of multijet events after the isolation requirement (194 \pm 18$)$ is obtained by multiplying this number with the isolation efficiency $(4.3 \pm 0.5) \%$, given by the ratio of isolated to all like-sign multijet events.

A second instrumental background arises from $Z / \gamma^{*} \rightarrow$ $\mu^{+} \mu^{-}$events in which the charge of one of the muons is misidentified. The first source of charge misidentification is due to fewer CFT layers at large $\eta$ and a consequent increase in the charge misidentification probability. The second source affects very high $p_{T}$ tracks for which the uncertainty on the measured curvature can cause charge misidentification. The charge misidentification rate is obtained by dividing the number of like-sign events $(S 1, S 2$, and $S 4$ ) by the number of events without the like-sign

TABLE I. The expected number of events for a signal with $M\left(H_{L}^{ \pm \pm}\right)=140 \mathrm{GeV} / c^{2}$ and background and the number of observed events after each selection step. The like-sign requirement is applied in the last two columns. The statistical and systematical uncertainties are combined in the table.

\begin{tabular}{lccccccc}
\hline \hline Selection & Preselection & Isolation & $\Delta \phi<2.5$ & Like sign & Third muon & & \\
& $S 1$ & $S 2$ & $S 3$ & $S 4$ & $S 5$ & $S 1 \& S 4$ & $S 2 \& S 4$ \\
\hline$Z / \gamma^{*} \rightarrow \mu^{+} \mu^{-}$ & $69181 \pm 4642$ & $58264 \pm 3910$ & $4936 \pm 333$ & $5.3 \pm 1.6$ & $<0.01$ & $84 \pm 24$ & $42 \pm 12$ \\
Multijet & $4492 \pm 120$ & $194 \pm 18$ & $18 \pm 2$ & $6.3 \pm 0.8$ & $0.2 \pm 0.1$ & $1620 \pm 34$ & $70 \pm 5$ \\
$Z / \gamma^{*} \rightarrow \tau^{+} \tau^{-}$ & $328 \pm 25$ & $269 \pm 21$ & $20 \pm 3$ & $<0.01$ & $<0.01$ & $3.2 \pm 1.3$ & $0.2 \pm 0.3$ \\
$t \bar{t}$ & $38 \pm 3$ & $20 \pm 1$ & $14 \pm 1$ & $0.03 \pm 0.01$ & $<0.01$ & $6.6 \pm 0.5$ & $<0.1$ \\
$W W$ & $40 \pm 3$ & $34 \pm 2$ & $20 \pm 1$ & $<0.01$ & $<0.01$ & $0.08 \pm 0.02$ & $0.04 \pm 0.01$ \\
$W Z$ & $19 \pm 1$ & $16 \pm 1$ & $11 \pm 1$ & $2.95 \pm 0.20$ & $1.62 \pm 0.11$ & $5.14 \pm 0.35$ & $4.25 \pm 0.29$ \\
$Z Z$ & $10 \pm 1$ & $9 \pm 1$ & $5 \pm 1$ & $0.63 \pm 0.05$ & $0.47 \pm 0.03$ & $1.12 \pm 0.08$ & $0.90 \pm 0.06$ \\
Total background & $7408 \pm 4644$ & $58806 \pm 3910$ & $5024 \pm 333$ & $15.2 \pm 18$ & $2.3 \pm 0.2$ & $1720 \pm 41$ & $117 \pm 13$ \\
Signal & $20.5 \pm 2.7$ & $18.5 \pm 2.4$ & $16.3 \pm 2.1$ & $11.6 \pm 1.5$ & $10.1 \pm 1.3$ & $16.4 \pm 2.2$ & $13.6 \pm 1.8$ \\
Data & 72974 & 58763 & 4558 & 16 & 3 & 1678 & 96 \\
\hline \hline
\end{tabular}


requirement ( $S 1$ and $S 2$ ) in the dimuon invariant mass region above $70 \mathrm{GeV} / c^{2}$, after subtracting the $\mathrm{SM}$ sources of background except $Z / \gamma^{*} \rightarrow \mu^{+} \mu^{-}$events from the data. This mass requirement removes most multijet background events in the low mass range. From these ratios, we determine the average probability for charge misidentification in data and $\mathrm{MC}$ simulations to be $P_{\text {data }}=(6.2 \pm$ $1.1) \times 10^{-4}$ and $P_{\mathrm{MC}}=(3.1 \pm 0.4) \times 10^{-4}$, respectively, assuming the multijet background is negligible. The uncertainties are statistical. A possible bias in such an estimate due to the potential presence of signal events in the sample has been estimated to be well below the assigned systematic uncertainty. Since the charge misidentification rate in MC simulations is underestimated, the ratio of $P_{\text {data }}$ to $P_{\mathrm{MC}}$ is taken as a correction equal to $2.0 \pm 0.4$. This ratio is applied to the $Z / \gamma^{*} \rightarrow \mu^{+} \mu^{-}$MC sample when estimating the like-sign contribution.

The distributions of dimuon invariant mass and $\Delta \phi$ after selection $S 1$ are shown in Figs. 1(a) and 1(b). The data are compared with the sum of the background contributions. For those events with more than one pair of muons fulfilling the selection criteria, the dimuon invariant mass and $\Delta \phi$ are calculated only for the pair with the highest individual momenta. The number of remaining events after each selection is shown in Table I. There is good agreement between data and the sum of the backgrounds. Figures 1(c) and $1(\mathrm{~d})$ show the dimuon invariant mass and $\Delta \phi$ distributions after the $S 1$ and $S 4$ requirements. The excess of events at $150 \mathrm{GeV} / c^{2}$ has a significance of less than $2.6 \sigma$. The last two columns of Table I give the individual likesign backgrounds after the various selection stages. This

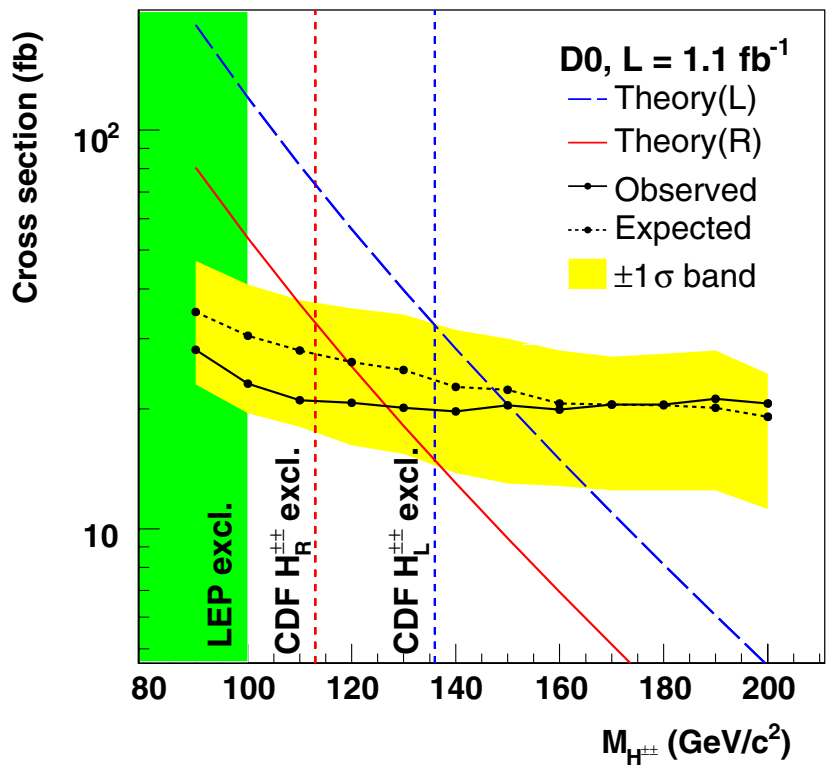

FIG. 2 (color online). The cross section limit as a function of the Higgs mass $M\left(H^{ \pm \pm}\right)$at the $95 \%$ C.L. The mass regions excluded by LEP and CDF are also shown. The $\pm 1 \sigma$ uncertainty on the expected limit is given by the shaded yellow band. demonstrates that the like-sign backgrounds are well understood.

After all five selection criteria, three data events remain, in good agreement with the SM background expectation of $2.3 \pm 0.2$ events. Total signal efficiencies are 32\%-34\% and are nearly independent of mass. The dimuon invariant mass and $\Delta \phi$ distributions for these events are compared to the sum of the backgrounds in Figs. 1(e) and 1(f).

Since no excess is observed, we use the dimuon invariant mass distribution in Fig. 1(e) to compute upper limits on the production cross section times the branching fraction as a function of $M\left(H^{ \pm \pm}\right)$using the $C L_{S}$ method [18] as implemented in the MCLIMIT program [19]. The expected rate for the signal as a function of $M\left(H^{ \pm \pm}\right)$is determined by the NLO cross section [5] and measured luminosity and corrected for the signal efficiency.

A number of systematic uncertainties on the signal and background are taken into account in the limit calculation. The uncertainties on the correction of the muon identification are $2 \%$ and $6 \%$ for the backgrounds and signal, respectively. The uncertainty on the isolation efficiency for the multijet background is $12 \%$. The $20 \%$ uncertainty on the correction for charge misidentification is included. The uncertainty on the luminosity for the signal is estimated to be $6.1 \%$ [20]. The uncertainty on the normalization using NNLO MC SM background production cross sections is taken to be 5\%. The parton distribution function uncertainties on the cross section for backgrounds are taken to be $4 \%$ [21].

The cross section limit as a function of $M\left(H^{ \pm \pm}\right)$is shown in Fig. 2 together with the theoretical cross section for left- and right-handed doubly charged Higgs bosons. Mass limits for other branching ratios can be obtained by scaling the theory cross section by the square of the branching ratio. At the $95 \%$ C.L., lower mass limits of $150 \mathrm{GeV} / c^{2}$ for left-handed and $127 \mathrm{GeV} / c^{2}$ for righthanded doubly charged Higgs bosons are obtained. This extends the previous mass limit [8] for a doubly charged Higgs boson decaying into muons.

We thank the staffs at Fermilab and collaborating institutions and acknowledge support from the DOE and NSF (USA); CEA and CNRS/IN2P3 (France); FASI, Rosatom, and RFBR (Russia); CNPq, FAPERJ, FAPESP, and FUNDUNESP (Brazil); DAE and DST (India); Colciencias (Colombia); CONACyT (Mexico); KRF and KOSEF (Korea); CONICET and UBACyT (Argentina); FOM (The Netherlands); STFC (United Kingdom); MSMT and GACR (Czech Republic); CRC Program, CFI, NSERC, and WestGrid Project (Canada); BMBF and DFG (Germany); SFI (Ireland); The Swedish Research Council (Sweden); CAS and CNSF (China); and the Alexander von Humboldt Foundation. 
${ }^{+}$Visitor from The University of Liverpool, Liverpool, United Kingdom.

${ }^{\ddagger}$ Visitor from ICN-UNAM, Mexico City, Mexico.

${ }^{\S}$ Visitor from II. Physikalisches Institut, Georg-AugustUniversity, Göttingen, Germany.

${ }^{\|}$Visitor from Helsinki Institute of Physics, Helsinki, Finland.

${ }^{\mathrm{I}}$ Visitor from Universität Zürich, Zürich, Switzerland.

**Deceased.

[1] J. C. Pati and A. Salam, Phys. Rev. D 10, 275 (1974); R. N. Mohapatra and J. C. Pati, Phys. Rev. D 11, 566 (1975); 11, 2558 (1975); G. Senjanovic and R. N. Mohapatra, Phys. Rev. D 12, 1502 (1975).

[2] G. B. Gelmini and M. Roncadelli, Phys. Lett. 99B, 411 (1981).

[3] N. Arkani-Hamed, A. G. Cohen, and H. Georgi, Phys. Lett. B 513, 232 (2001).

[4] J.F. Gunion, H. E. Haber, G. Kane, and S. Dawson, The Higgs Hunter's Guide (Addison-Wesley, Redwood City, CA, 1989).

[5] M. Mühlleitner and M. Spira, Phys. Rev. D 68, 117701 (2003).

[6] G. Abbiendi et al. (OPAL Collaboration), Phys. Lett. B 526, 221 (2002); P. D. Acton et al. (OPAL Collaboration), Phys. Lett. B 295, 347 (1992); P. Achard et al. (L3 Collaboration), Phys. Lett. B 576, 18 (2003); J. Abdallah et al. (DELPHI Collaboration), Phys. Lett. B 552, 127 (2003).
[7] V. M. Abazov et al. (D0 Collaboration), Phys. Rev. Lett. 93, 141801 (2004).

[8] D. Acosta et al. (CDF Collaboration), Phys. Rev. Lett. 93, 221802 (2004).

[9] V. M. Abazov et al. (D0 Collaboration), Nucl. Instrum. Methods Phys. Res., Sect. A 565, 463 (2006).

[10] The pseudorapidity is defined as $\eta=-\ln \left[\tan \left(\frac{\theta}{2}\right)\right]$ as a function of the polar angle $\theta$.

[11] S. Abachi et al. (D0 Collaboration), Nucl. Instrum. Methods Phys. Res., Sect. A 338, 185 (1994).

[12] V. M. Abazov et al., Nucl. Instrum. Methods Phys. Res., Sect. A 552, 372 (2005).

[13] T. Sjöstrand et al., Comput. Phys. Commun. 135, 238 (2001).

[14] R. Hamberg, W. L. van Neerven, and T. Matsuura, Nucl. Phys. B359, 343 (1991); B644, 403(E) (2002).

[15] N. Kidonakis and R. Vogt, Int. J. Mod. Phys. A 20, 3171 (2005).

[16] J. M. Campbell and R. K. Ellis, Phys. Rev. D 60, 113006 (1999); http://mcfm.fnal.gov/.

[17] R. Brun and F. Carminati, CERN Program Library Long Writeup No. W5013, 1993.

[18] A. Read, J. Phys. G 28, 2693 (2002).

[19] T. Junk, Nucl. Instrum. Methods Phys. Res., Sect. A 434, 435 (1999).

[20] T. Andeen et al., FERMILAB Report No. FERMILABTM-2365, 2007.

[21] J. Pumplin et al., J. High Energy Phys. 07 (2002) 12. 